\title{
Standards Based Household Load Control Prototype
}

\author{
Kaium Uz Zaman Mollah, Momen Bahadornejad and Nirmal-Kumar C. Nair \\ Department of Electrical and Computer Engineering \\ The University of Auckland \\ Auckland, New Zealand
}

\begin{abstract}
In this paper a platform is developed to facilitate the control of household loads using communication protocols relevant to home automation and substation automation systems. Protection IEDs that support IEC 61850 standard based protocol, universal secondary test gear for relays and a protocol converter are used to realize the protection/automation aspects for substation automation. The retrofitting of the latest relay IEDs with the existing control center software has also been demonstrated. In the home automation space, the available commercial Programmable Logic Controller (PLC) and IEDs are used to develop the platform. The automation load control scheme is programmed based on IEC 61131 standards using structural language and is implemented through EtherCAT protocol. The PLC uses the IEC 61850 server to communicate and gather raw data form the power measurement terminal. The developed platform establishes different aspects of automation and standard based communication system identified the Smart Grid framework. This prototype integrates two different vendors' intelligent electronic devices and implements a smart load shedding algorithm which controls load in real time.
\end{abstract}

Keywords- Automation, smart grid, load shedding, Programmable Logic Control, Intelligent Electronic Devices (IED).

\section{INTRODUCTION}

The conventional power system is shifting onto smart grid (SG) to meet the various automation and integration needs. The SG consists of the latest technologies and is capable of bidirectional communication between utilities and consumers. Advanced Metering Infrastructure (AMI) comes along with SGs [1]-[2]. AMI is a system that measures and analyzes the energy usage and communicates through a range of media with smart meters. This interconnected advanced network will improve the system reliability and security [3].

In the era of rapid popularity of SG, Intelligent Electronics Devices (IED) and AMI has led to a diverse building/ home automation systems and substation automation to be evolved [4]-[6]. With the increased growth of SG infrastructure and automation systems, there is a need of interoperability between various devices and networks which are provided by a wide range of vendors. Therefore, there has been a development of diverse standards. The abbreviation of SG Friendly Devices defines the devices that can be automatically controlled via SG network [7].

At present the load tripping during under voltage/frequency events is done by triggering the feeders as depicted in Fig.1. In near future the power consumer premises will be equipped with emerging IEDs, which will monitor dynamic grid data at all the times [1]. If IEDs detect an increasing power demand or an under frequency/voltage event, then they will control electrical appliances in an individual house or a building. Thus, the under voltage issue will be mitigated locally. This helps in relieving the increased stress on the power grid before the issue has any major effect on the main grid system. Thus it ensures the power grid to maintain a stable and healthy condition in the long term [8]-[9]. Therefore this is a smarter way to control loads, balance the power generation to the power consumption and to protect the power grid. The SG automation load control and priority load shedding (LS) are still on a research stage and there is a need to new algorithms to improve the system reliability and security.

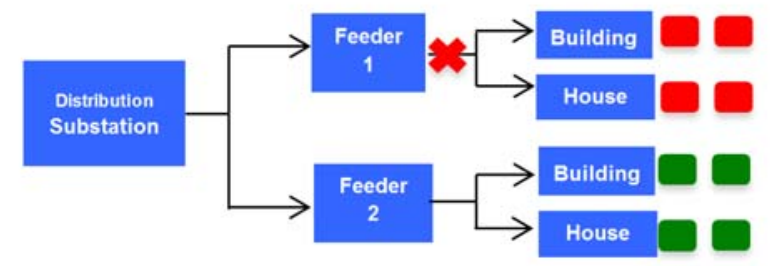

Fig. 1 Conventional load control method

In this paper an intelligent automation platform is developed to facilitate the control of household loads. The substation automation (SA) part of the laboratory test bench presented in this paper is a combination of virtual transmission system with real IEDs and other equipment [2]-[3][5]. The standards communication protocol IEC 61850 has been used for this implementation using GOOSE messaging and the Continuous Function Chart (CFC) programs which is a proprietary device specific programming option. The data collected by IEDs can be broadly categorized as operational data, which are in the form of analog and digital values, and non-operational data which are essentially waveform data.

In the home area (HA) part of the test bench, PLC along with IEC 61850 standards are used to implement the load control algorithm. TwinCAT software was used to write the LS algorithm. The power measurement terminal of PLC samples the relevant dynamic electrical data [10, 11]. This measured data is then compared against the set values in the algorithm (TwinCAT software) to recognize under voltage (UV) events. A load shedding algorithm is implemented using the real time data [12]. The preferences for LS are set by assigning each load with a specific priority number. The load with the lowest priority is shed first. When the controller needs to fulfill the power targets, it starts by turning off the specific load. If this load does not have enough power to 
achieve the necessary voltage value, the controller tries the second load on the list and so on.

Section II of this report describes the methodology and algorithm of load control scheme. This is followed by the section III which discusses the automation platform. Section IV of the report outlines the simulations and results. The final section is devoted to the conclusions to summaries the findings.

\section{PLATFORM OVERVIEW}

The platform architecture overview is illustrated in Fig 2. In the first part of the prototype a communication platform between substation IEDs to LV level IEDs was designed. The test bench uses GOOSE protocol which works on publishersubscriber mechanism. GOOSE data are grouped in packets and transmitted within time period below four milliseconds to assure protective relaying and reliability. Twincat telecontrol software is used to set different IEC61850 parameters. After setting the IEC61850 parameters, device description file (ICD file) and PLC code file can be exported. The ICD file is then imported in the DigS4 software which is used to configure the Siemens relays. On the contrary, the PLC code file is imported in PLC control programme thus establishing the communication between PLC and Relay. Whole PLC-Relay communication sequence is depicted in Fig. 3.

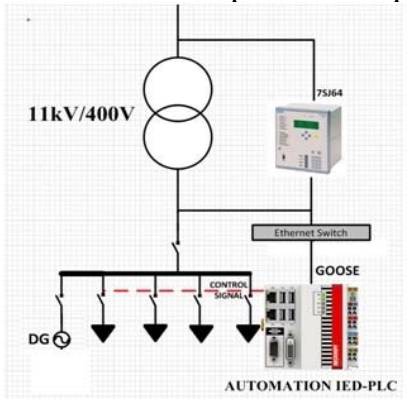

Fig. 2 Load Control Architecture

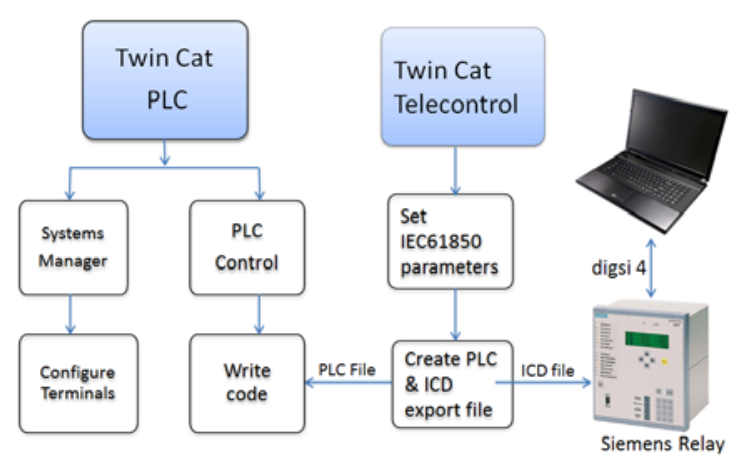

Fig. 3 IEC61850 Communication Sequence

The second part is designed to control the LV level load by using the smarter PLC logic with real time data. This control part uses PLC-Relays communication (IEC61850 protocol) and performs voltage based LS in the LV level. The communication data is transferred on Ethernet. Twincat software is used to programme the PLC. The code to programme the PLC is written in PLC control window and
PLC terminals are configured using system's manager of TwinCAT software.

\section{SERVER-CLIENT COMMUNICATION}

The test bench uses SIEMENS SIPROTEC 7SJ64 multifunction protection relay. The one node architecture is composed of IED SIPROTEC 7SJ64 at substation level and PLC at the LV level and uses IEC61850 server for communication purpose. The IEC61850 server also known as TwinCat telecontrol was installed on the PC to declare all the important aspects of IEC61850 standard such as logical devices, nodes and data attributes and then a PLC export file can generated. Fig. 4 shows the sample code where the client (SIPROTEC 7SJ64) IP address is declared for communication purpose.

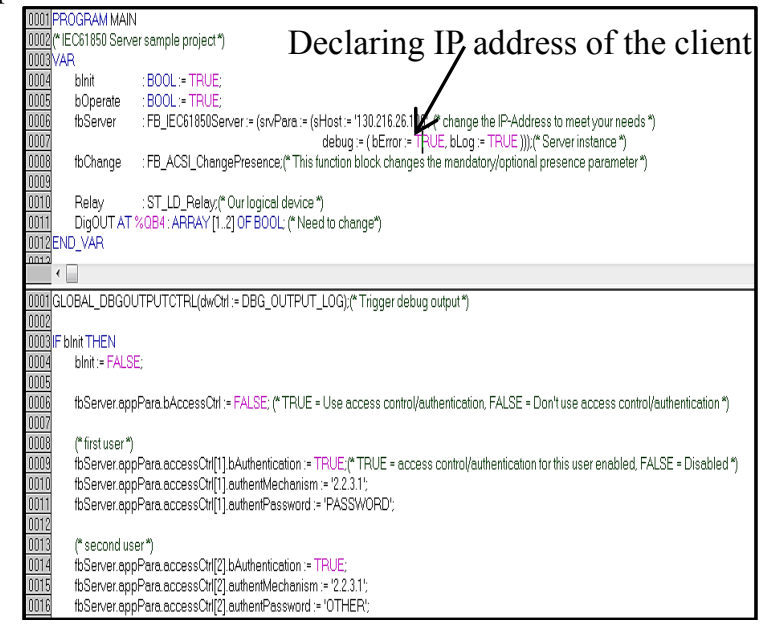

Fig. 4 Server-Client Communication

The IEC61850 server (Twin Cat Telecontrol) software window is divided into 5 important parts. The window is shown in Fig. 5 and parts are explained as following:

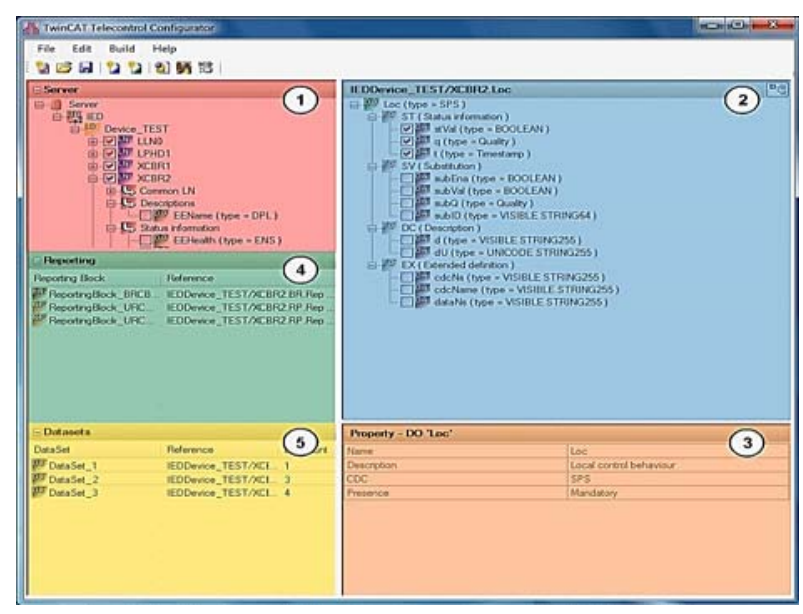

Fig. 5 Telecontrol Server Window

1) Server Configuration: It contains all the information regarding logical devices and logical nodes.

2) Data Object Configuration: All the information regarding data attributes selected in the server configuration. 
3) Object properties: All the properties linked with the selected object,

4) Reporting: The reporting blocks buffered and buffered are listed in this section

5) Data Sets: Created dat sets can be seen in this section

\section{PROTOTYPE COMMISSIONING}

This section discusses in detail the developed automation experimental setup and necessary software required for implementing the load control algorithm. The prototype commissioning and the automation visualization are also briefly described.

\section{A. Hardware and Experimental Setup}

The major required hardware for designing the automation platform is tabulated in Table I. The prototype parameters are represented by serial ID number1-10.

TABLE I: MAJOR PARAMETERS OF THE PROTOTYPE

\begin{tabular}{|c|c|c|}
\hline ID & Description & Parameter \\
\hline 1 & Three Phase AC Power Supply & 0 -300Vrms \\
\hline 2 & DC Power supply to PLC & $24 \mathrm{~V}$ \\
\hline 3 & Variable Resistive loads & $1 \mathrm{~K}$ \\
\hline 4 & 3-phase current transformer(CT) & $5 \mathrm{~A} / 1 \mathrm{~A}$ \\
\hline 5 & Fast Acting Fuses & $2 \mathrm{~A}$ \\
\hline 6 & Embedded PC & $\begin{array}{c}\text { CX5020 PLC } \\
\text { Controller }\end{array}$ \\
\hline 7 & EL3773 Terminal & $\begin{array}{c}\text { 3-phase Power } \\
\text { monitoring }\end{array}$ \\
\hline 8 & EL2622 Terminals & 0-230V Output \\
\hline 9 & Communication Protocol & Ether Cat \\
\hline 10 & Controllable Loads & 4 X Lamps \\
\hline 11 & Automation Visualization & TwinCAT software \\
\hline
\end{tabular}

A 3-phase AC power supply unit was used to supply the power to the system. Phase 1 was set up at $0^{\circ}$, phase 2 at $120^{\circ}$ and phase 3 at $240^{\circ}$. Fig. 6 shows the developed automation platform. The number 1 is represented three phase powersupply unit and number 3 is represented variable resistive load and so on. The developed prototype was secured in a safety box that consists of the PLC and the I/O terminals. Two EL2622 output terminals were used to control the loads which were connected to each phase. The EtherCAT EL3773 power monitoring oversampling terminal was used to monitoring terminal for state monitoring of a 3-phase AC voltage system.

The load control priority and the amount of load for each phase are represented in Table II. Four loads (L1, L2, L3, and L4) connected to three phase supply were selected. The preferences were set by assigning each load with a given priority. It is clear from the table that Load 1 is given the least priority and load-4 is given the highest priority. The load with the lowest priority was shed first.

When the controller needs to fulfill the power targets, the controller starts by turning off load-1. If this load does not shed enough power to achieve the necessary voltage threshold, then the controller shed a second load in the list and so on.

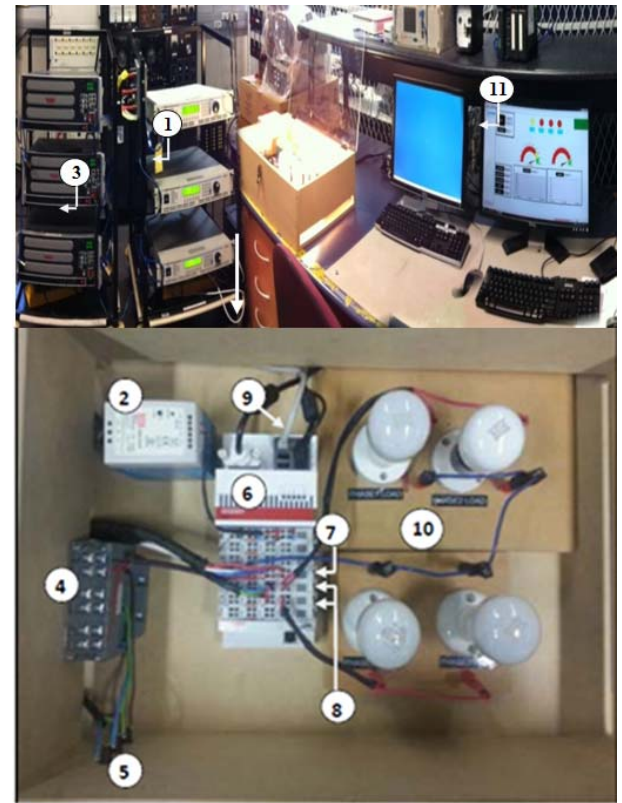

Fig. 6 Developed prototype and the safety box

TABLE II: LOADS PARAMETERS

\begin{tabular}{|c|c|c|c|c|}
\hline Load & Priority & $\begin{array}{c}\text { Rated } \\
\text { output(watts) }\end{array}$ & Phase & Total \\
\hline L1 & 1 & 100 & 1 & $100 \mathrm{~W}$ \\
\hline L2 & 2 & 100 & 1 & $100 \mathrm{~W}$ \\
\hline L3 & 3 & 100 & 2 & $100 \mathrm{~W}$ \\
\hline L4 & 4 & 25 & 3 & $25 \mathrm{~W}$ \\
\hline \multicolumn{5}{|c|}{ Total } \\
\hline
\end{tabular}

In order to measure the electrical data from the distribution Generation new EL3773 power measurement terminal was bought. After properly understanding the wiring connection and working of this terminal and it was able to install it in PLC. This terminal has the capability to measure 10,000 samples per second so the values can be calculated very precisely as shown in Fig. 7.

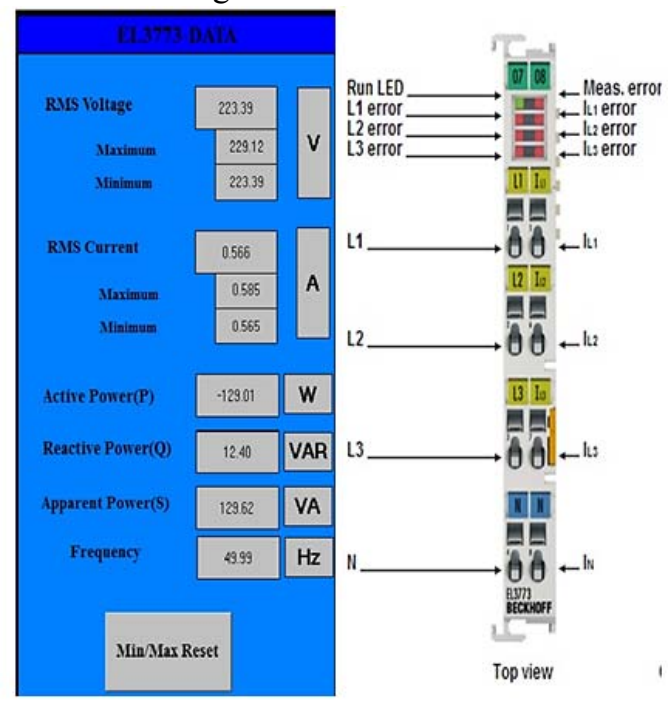

Fig. 7 EL3773-Power Measurement Terminal 
The number of calculations to perform before the actual measured value is displayed can be set in the TwinCAT Programme used to configure this terminal. For example if a large number is set then it will take extra time to display the present value. In normal case 100 calculations are set in the program as can be seen in Fig. 8.

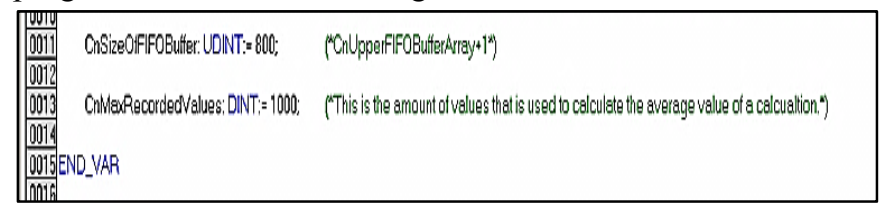

Fig. 8 EL3773 Configuring programme

\section{B. Software}

The SIEMENS software DIGSI V4.86 has been used to configure the SA IEDs, program the logic sequences and to make GOOSE communication. Besides, all devices are connected to the network via Fiber or Ethernet ports and have their own IP address to provide the communication [5].

The commercial PLC is used to develop home automation (HA) platform. The PLCs generally are widespread in industrial level and can perform an advanced automation processes. The PLC is an embedded PC with Windows CE. This device is capable of several secure communication standards. They are EtherCAT, IEC61131, IEC 61850, Distributed Network Protocol (DNP3) and Modbus. A PLC is typically consisting with a power supply, controller program and working memories, I/O terminals and a central control unit [7].

TwinCAT PLC is programmed based on IEC 61131-3 programming languages with convenient editors and a fast, effective compiler. It was used to develop HA algorithm using structured text [13]. TwinCAT provides two different environments; PLC control and systems manager. The automation algorithm was developed using the PLC control environment and the systems manager was used for configuring different input output terminals to the PLC.

TwinCAT PLC IEC61850 server is used to communicate the data and send control commands in the PLC. The communication stack developed is based on the MMS protocol. PLC is capable of communicating with EtherCAT terminals in 40 microseconds. Fig. 9 shows the PLC control environment (left side) and Systems Manager (right side).

\section{Prototype Commissioning}

A constant 24 VDC power supply was used to power the PLC. The maximum current rating for power measurement terminal is 1 ampere. Consequently, CTs with a primary to secondary ratio of 5 to 1 were used to protect from the higher currents. As the current reading was taken from the secondary side of the transformer, thus a ratio factor was used in the developed algorithm to obtain the actual current flow in the circuit. For example, if the measured value of current was 1A then it was multiplied with 5 in TwinCAT software to get actual value $(1 \mathrm{~A} * 5=5 \mathrm{~A})$. Three $2 \mathrm{~A}$ fuses were installed in each phase before the primary side of the $\mathrm{CT}$ for providing over current protection to the power measurement terminal.

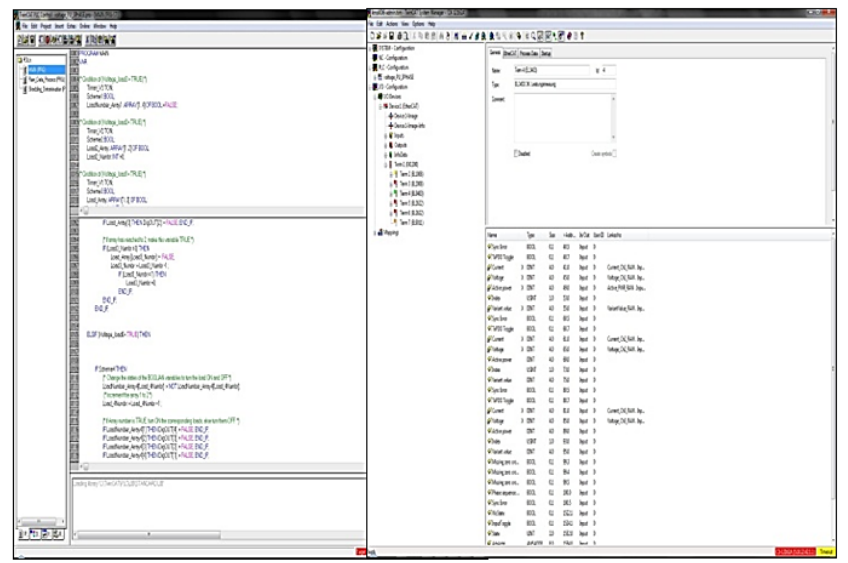

Fig. 9 Software environments

Three variable resistive loads were used to control the amount of current flow in the circuit. A wooden safety box was used to provide protection against high voltage. All the major components such are the PLC, 3-phase CT, three 2A fuses and loads(incandescent bulbs) associated with high voltage were placed inside the safety box. A high voltage three-phase switch was also installed outside the safety box to turn off the power supply during faults and a transparent glass top was fitted on the safety box to provide extra security.

The system manager environment available in TwinCAT software was used to configure I/O terminals of the PLC through IEC61850 standard. The communication and control of the $\mathrm{I} / \mathrm{O}$ terminals were established using the EtherCAT protocol. The overall circuit diagram including connected loads is shown in Fig. 10.

To configure the PLC and the I/O terminals, the initial step was to add the I/O terminals to the TwinCAT system manager software. Then download the developed PLC logic onto the PLC through the TwinCAT systems manager.

Finally, the created input and output variables on the PLC logic files were mapped to the corresponding terminal variables. The light emitting diodes (LEDs) on the top of the power measurement terminal indicate voltage and current states of each phase. The high voltage output terminal also consists of LEDs that represent the states of connected loads.

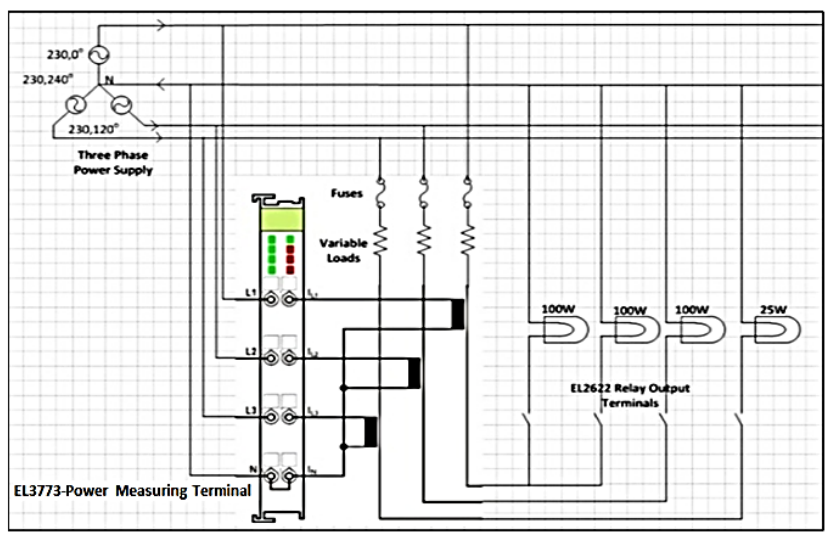

Fig. 10 PLC commissioning and load wiring 


\section{Automation Visualization}

The visualisation was interactive (can be controlled using smart phone/tablet) and was built to automate the substation functions. The visualization was developed for low voltage side and the Graphical User Interface of the automation platform was developed using the TwinCAT software as shown in Fig.11. The system is assumed to have two statuses which are normal and contingency. During the normal case (indicated by blinking Normal box) the residential loads will be powered from the grid. In case of feeder fault or contingency (indicated by blinking Contingency box) the monitoring IED (Siemens relay) will receive signal from the medium voltage station. After receiving the signal monitoring IED will indicate to PLC via goose communication.

The grid connection will be disconnected (islanded) and will be powered through Distributed generation. The power supply (device ID: 1) shown in Fig. 6 is used as distributed generation. The power measurement terminal EL3773 gathers all the relevant electrical data from DG that is displayed as shown on the left side of visualization. This will include the minimum and maximum values that have been recorded which can be reset as well. All the icons in the visualization are interactive so it can be controlled using a smart phone or other similar electronic device. The visualization can be operated in three different modes as shown on top left of window in figure 11 and are explained below.

Automatic: LS depending upon the voltage values of DG.

Manual: LS by manual clicking on each device

Emergency: All the loads will be turned off at the same time.
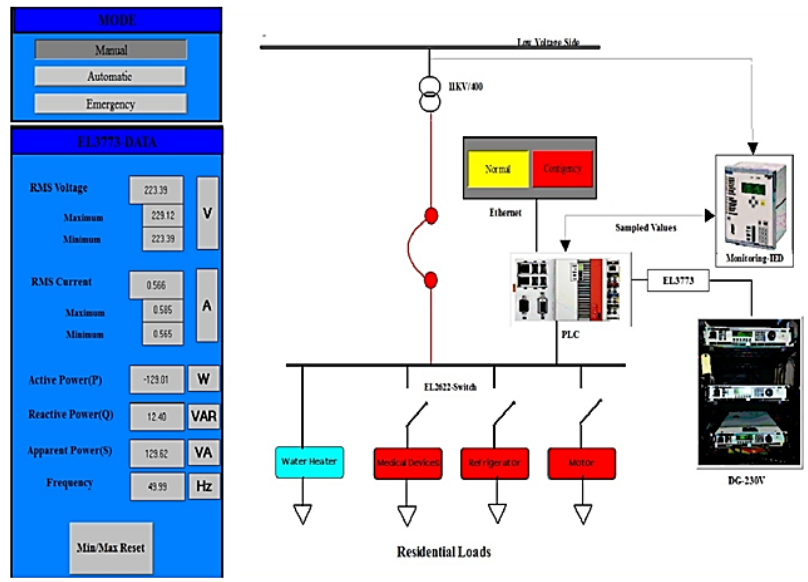

Fig. 11 Developed Automation Visualization.

The electrical data gathered by EL3773 terminal contains following information.

- System status indicators (blinking normal or contingency LED)

- Measured present, minimum and maximum values of voltage and current with reset option.

- Status of each connected loads

- Active, reactive and apparent power consumption of phase

\section{CASE STUDY: INTELLIGENT LOAD SHEDDING SCHEME}

In current practice automatic LS schemes are operated at the medium voltage (MV) level. The emergence of SG technologies that are cheaper has created more control options that can be implemented for distribution LV network operation. This section illustrates a smarter LS scheme at the LV level using the real data.

To avoid voltage collapses UV based LS schemes have been integrated to the power system as the last solution to prevent power system instability. AUVLS is used when the voltage goes down to a predefined limit. At this limit an amount of load will be shed so the voltage can stay in an acceptable voltage range. The different steps involved in LS are [10]:

- Determination of the amount of load to be shed,

- Selection of loads to be dropped,

- Determination of time steps of load shedding (by steps to avoid over shedding),

- Determination of voltage level at which shedding begins.

The LS sequence is shown in Fig.12. The voltage based LS IED is triggered when the voltage drops to the setting. The loads in the consumer's premises are classified according to their importance (critical and non-critical loads). The critical loads such as medical devices are turned off at last.

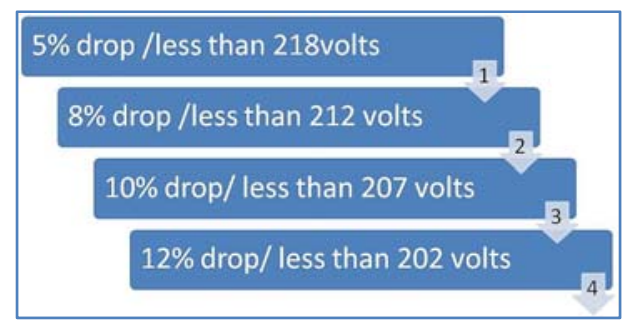

Fig. 12 Voltage based shedding sequence

\section{A. Load Shedding Algorithm}

The substation IED SIPROTEC 7SJ64 sends a signal to the PLC server representing state of the grid. Depending upon the state of the signal the system can be islanded and fed through Distributed generation as shown in Fig. 2. Based on the voltage value of DG loads can be controlled. This method includes the following steps:

Step1: Measure voltage at receiving end of each node using EL3773 terminal

Step2: After the system contingency, recalculate voltage magnitude again at the receiving end of each node.

Step3: Compare the node voltage (p.u.) with the normal range of voltage, if the voltage goes below 0.92 p.u. then go to step 4. The LS scheme would not operate if the voltage stays within the setting limits ( 0.92 p.u.-1 p.u.).

Step4: Loads are tripped according to priorities in Table II.

Step5: The relay is activated after 1 second and curtails a fixed amount of load consistently until a load/supply balance is attained. Objective of 1 second delay was to check if the system has recovered from under voltage event and also to avoid unwanted tripping of loads. The four LS steps are the shedding load 1 to load 4 with a delay of $1 \mathrm{~s}$ when per unit 
voltage reduces to $0.92,0.90,0.88$, and 0.86 respectively.

In this section, under voltage scenario is considered. The LS event begins with the measurement of three phase supply by the power measurement terminal of PLC. Fig. 13 shows the simulations result that was carried out on phase 1 . It is wise to admit that voltage was reduced manually to create the scenarios.

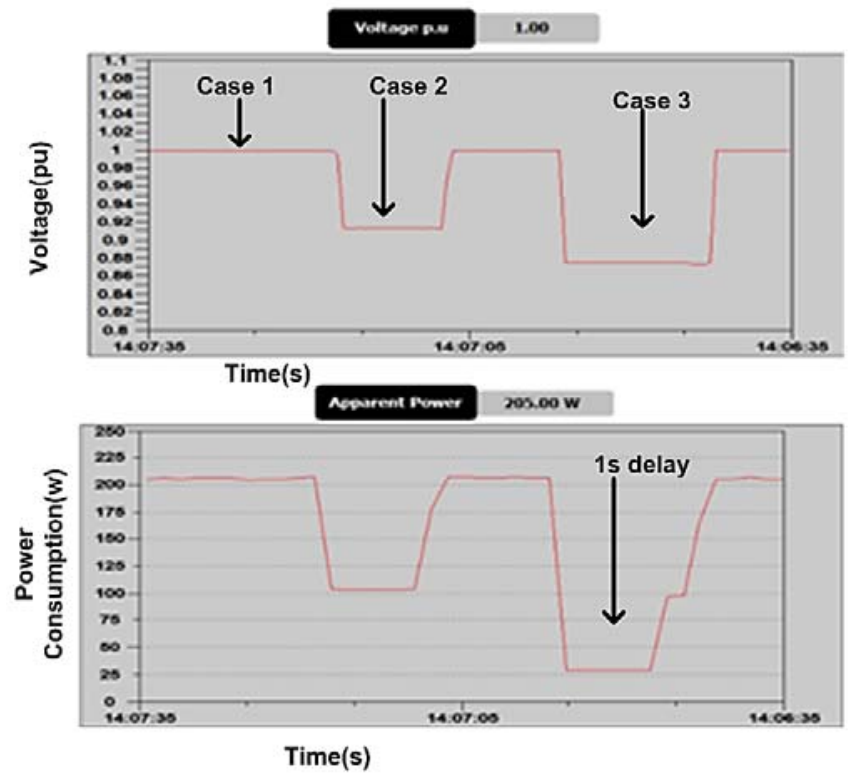

Fig. 13 Experimental results

Case 1: It represents the normal operation without any disturbances and voltage magnitude stay at $1 \mathrm{pu}$.

Case 2: It shows when the voltage magnitude reduces to 0.92 p.u. Since the controller dynamically monitors the voltage magnitude, it recognizes this change and trips the first load connected to phase1. Therefore, a power reduction of $100 \mathrm{~W}$ can be seen in the corresponding power graph.

Case 3: It is an extension of case 2 where the voltage magnitude reduces to $0.88 \mathrm{p}$.u. Two successive loads were disconnected with a time delay of 1 second and the reduction in power can be seen in the following graph.

\section{CONCLUSIONS}

A laboratory version of standards based automation load control platform has been developed. Automation load control scheme for different operating environments was implemented using SG friendly devices. The developed automation prototype performs load control methods in response to voltage variation. The developed prototype uses a PLC, I/O terminals; three phase current transformers, fuses, four loads (incandescent bulbs), three variable resistive loads and three phase power supply. The experimental result demonstrates that the developed controller is able to control the connected loads. It can be used to mitigate the under voltage events locally rather than turning off the feeder that creates blackouts.
Due to the vastness of the LV network, the financial investment for wide-spread deployment is likely to be minimized making the suggested prototype an attractive option. Although, the cost varies for different types and sizes of residential properties, integration of other devices such as smart meters and ripple relays can reduce the development and installation cost. This is an ongoing research investigation.

\section{ACKNOWLEDGMENT}

The second and the third author acknowledge the funding provided by New Zealand Ministry of Business Innovation and Employment (MBIE) for the GREEN Grid project for their time in this work. The GREEN Grid project is a joint project led by the University of Canterbury with the University of Auckland's Power System Group and the University of Otago's Centre for Sustainability, Food and Agriculture, and with a number of electricity industry partners. The project, officially titled "Renewable Energy and the Smart Grid" will contribute to a future New Zealand with greater renewable generation and improved energy security through new ways to integrate renewable generation into the electricity network.

The authors would also like to thank Jaskaran Singh, a summer research student, for his contribution in this work.

\section{REFERENCES}

[1] J.Wang, V.C.M.Leung, "A Survey of Technical Requirements and Consumer Applicaiton Standards for IP-based Smart Grid AMI Network"

[2] Y.Wang, D.Raun, D.Gu, "Analysis of Smart Grid Security Standards"

[3] S.Sucic, B.Bony, L.Guise, "Standards-compliant Event-driven SOA for Semantic-enabled Smart Grid Automation: Evaluating IEC 61850 and DPWS Integration" - ICIT 2012

[4] L.Cheng, "Study and Application of DNP3.0 in SCADA Systems" International Coference on Electronic and Mechanical Engineering and Information Technology 2011

[5] D. Milhau, et al., "Laboratory test bench demonstrating smart load shedding schemes," in Power System Technology (POWERCON), 2012 IEEE International Conference on, 2012, pp. 1-6.

[6] Apostopolov A., "Muti-agent Systems and IEC 61850" IEEE PES Multiagent System Taskforce Panel Paper-Meeting 2006.

[7] A.Pereira, C.Lima, J.F.Martins, "The use of IEC 61131-3 to enhance PLC control and Matlab/Simulink process simulations".

[8] Waxman, R., Design Automation Standards Development. Reliability, IEEE Transactions on, 1987. R-36(5): p. 507-513.

[9] M.Kanabar, I.volob, D.McGinn, "Reviewing Smart Grid Standards for Protection, Control and Monitoring Applications"- 2011

[10] http://www.beckhoff.co.nz/english.asp?twincat/twincat_plc_iec_61850_ server.htm

[11] Cosse, R.E., Jr., J.E. Bowen, and S.H. Kerr, "Secondary selective system residual bus transfer - a modern application approach", Industry Applications, IEEE Transactions on, 2005. 41(1): p. 112-119.

[12] K. Mollah, et al., "Automatic under-voltage load shedding: A systematic review," in Power and Energy Society General Meeting, 2012 IEEE, 2012, pp. 1-7.

[13] ftp://ftp.beckhoffautomation.com/TwinCAT_Manual/TwinCAT\%202\% 20Manual\%20v3.0.1.pdf 